\title{
FPGAs in Manufacturing (Product Inspection System): Presence/Absence Detection
}

\author{
Evin Ramadeen and Marcus Lloyde George \\ UG Student, Department of E\&C Eng, University of the West Indies, St. \\ Augustine, Trinidad and Tobago Dept. Electrical and Computer Engineering \\ University of the West Indies St. Augustine, Trinidad and Tobago \\ Marcus.George@sta.uwi.edu
}

\begin{abstract}
This paper presents a conceptual design for an object presencelabsence detection system that will be used in the Manufacturing Industry using a Field Programmable Gate Array (FPGA). We will focus on the development of Automated Visual Inspection tools based on FPGAs.
\end{abstract}

Keywords: Background Subtraction, Motion Detection, Presence-Absence, FPGA, Image Processing, Spartan-3, Visual Inspection in Industry

\section{Introduction}

Product Inspection is an extremely important part of the manufacturing process to ensure the quality and completeness of the product. As technology improves and our dependency on electronics, specifically automation, increases, the need for the devices to detect the presence or absence of objects quickly is of great importance. In industries, many different methods of presence/absence detection is used dependent on the amount of detail required. For applications such as juice packaging, a simple laser detection method can be used to sense the presence or absence of the juice carton before filling commences. In other industries, Automated Visual Inspection is used to ensure that all components are present in the product being manufactured. The basis behind this Visual Inspection is the ability of a device to capture an image of the component, subsequently analyse the image and determine if the product is present and complete. These systems are designed to detect defects and faults in the manufacturing process of the process. The inspection task and technique varies according to the type of the product.

It is a well-documented fact that real-time computer vision is a computationally expensive task due to the fact that all images need to be subjected to many processes regardless of its size. In an attempt to minimize the amount of time the system takes to determine whether or not an object is present, thereby increasing the overall productivity of the plant, an algorithm that can satisfactorily handle the tasks is preferred. As such, the background subtraction based algorithm [1] is suggested for this particular object detection method as it is not a computationally expensive algorithm and it also presents suitably high performance in terms of its accuracy. In this paper, we propose the implementation of an efficient object presence/absence detection system on FPGA that can be employed in the manufacturing industry. This implementation is aimed at providing a high performance system that utilizes low power.

The manufacturing industry, being as important as it is, requires the systems it utilizes to be reliable. As such it is essential that the processing time per frame be as low as possible [2]. Due to this, it is important that the techniques being employed are as simple and efficient as possible, hence, the use of the background subtraction technique. This 
technique allows moving objects to be detected by taking the point-by-point absolute difference of the current image and a background image which must be acquired when there are no moving objects in the scene. Essentially, the concept behind the background subtraction approach is to find the mobbing objects by subtracting the current frame (pixel by pixel for increased accuracy) from a reference image called the background [1]. Seeing that in the manufacturing industry, the objects themselves are being continuously moved whilst the background remains unchanged, this method can be implemented. Another algorithm which could be implemented is known as the temporal difference algorithm. In the case of the temporal difference algorithm, instead of the current frame being subtracted from a reference background image, the current frame is subtracted from the previous frame which is used as a reference image. This is also a low cost algorithm but given the general operational conditions in the manufacturing industry, the background subtraction method is much more suitable as it offers the same level of accuracy whilst requiring less computational power as the reference image remains constant[5].

\subsection{About FPGA}

Field Programmable Gate Arrays (FPGAs) are a form of reconfigurable computing technology. Reconfigurable computers are processors which can be programmed with a design and then subsequently reprogrammed/reconfigured with a new design a virtually limitless number of times. Due to this, it can in some ways be seen as ideally suited for video processing. This type of architecture thereby allows for a large variety of logic designs, which are dependent on the processors resources, which can be interchanged for a new design as soon as the device can be reprogrammed. Generally, the hardware language used with FPGAs is VHDL[3].

\subsection{About VHDL}

VHDL is an acronym for "Very High Speed Integrated Circuit Hardware Description Language. VHDL is a hardware description language which can be used to model digital systems at many levels of abstraction which range from the algorithmic level to the gate level[3].

\section{Methodology}

Given that this system is intended to be implemented in the manufacturing industry, it becomes possible to apply stringent time restrictions on certain operations that the system is required to adhere to in order for accurate results to be produced. These restrictions include the average amount of time that is expected to pass between one object leaving and the next passing. That is, the time between two successive objects or items that is being manufactured. By being aware of this time, it becomes possible to minimize the amount of processing that the FPGA is required to do by allowing it to capture images within a certain time frame only instead of constantly. This will greatly reduce the overall time the FPGA takes process whether or not the object is present. By being able to do determine if an object is present in a short amount of time, the FPGA will then be available to perform other operations. These operations may include but will not be limited to the FPGA being used to determine what the object is that was captured in the previous frame. This additional application may be very pertinent to industries that produce multiple types of objects. By being able to determine what the object is that has been detected, it then becomes easy for the FPGA to be used to sort the different types of objects being produced as well. This paper will not consider these additional features and 
will focus on using the FPGA as a tool to determine the Presence/Absence of objects only. For the purposes of this system, it is assumed that a minimum delay of 5 seconds and a maximum delay of 10 seconds is expected between two successive objects. This means that the system is only required to capture images for the last 5 seconds of the expected time and if no object is detected within that time period, it can be considered as absent and a notification will be sent to the supervisor in the control room. By simply notifying the supervisor, the system allows the plant to continue normal operation whilst the supervisor or higher level system controller can take the required action to handle the absence of the particular object. The major drawback to the implementation of this system using the background subtraction method is the fact that it is highly dependent on some key factors. These are:

- The lighting of the plant needs to be of sufficiently high quality for the objects to be distinguishable from the background.

- The background behind the objects, which makes up the reference frame, needs to be a constant background with no changes being made during the operation of the system. This is to ensure that the system will not detect a change in the background and produce any incorrect results.

The process to determine the presence/absence of an object can be broken down into the following steps:

1) Capture Image of background and store in memory.

2) Capture video for the last 5 seconds of the 10 second allowed time period.

3) As images are captured, extract the frames.

4) Using the absolute difference algorithm, take the absolute difference of the current image/frame and the reference background image.

5) Select a proper threshold level to convert the subtracted image into a binary image.

6) Perform convolution on the proposed $3 \mathrm{X} 3$ masks with the binary image to filter out the noise.

This process is further illustrated in figure 1 below:

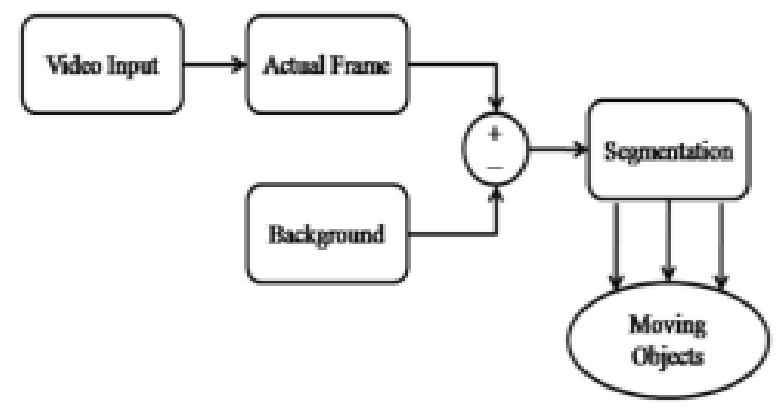

Figure 1. The Background Subtraction algorithm for Object Detection [4]

As is described in figure 1, this method states whether or not moving objects exists in the frame. This approach is applicable for this system implementation as the presence of a moving object signifies that an object is indeed present and subsequently, if no moving object is picked up, then the object is deemed absent. 
If we were to describe this background subtraction method mathematically, we would use the following equation:

$d(x, y, t)=1$ if $f(x, y, t)-B(x, y)>T_{d}$

(1)

$$
d(x, y, t)=0 \text { otherwise }
$$

Where $T_{d}$ is the predetermined threshold, $f(x, y, t)$ is representative of the image frame at some time, $t$, and $\mathrm{B}(\mathrm{x}, \mathrm{Y})$ is the reference background image. When the analysis is conducted, all pixels in the image represented by $\mathrm{d}(\mathrm{x}, \mathrm{y}, \mathrm{t})$ with the value 1 are considered as moving objects.

If this system was being created as an object recognition system, after the process described above recorded the presence of a moving object, it would be possible to verify the size and shape of the object and whether or not it is indeed the object that should be there by comparing the collection of pixels that registered as a 1 against an image which contains the expected object. If no differences are registered then it can be stated that the object is indeed the correct one [5]. For the purposes of this system however, such detail is not required and as such, this improvement shall not be discussed further.

Now, after extensive research, it was found that there are different ways to include processors inside the Xilinx FPGA for System-on-a-Chip (SoC). For the purposes of this system, it is proposed that we use the Xilinx 32-bit MicroBlaze processor as it offers a lot of flexibility [2].

\subsection{Related Work}

The ability of a system to conduct Presence/absence detection in itself is a very useful and ultimately beneficial tool in many different situations. As such, with improvements in technology, work is being done in this field with a great deal of regularity. As was previously stated, there are numerous different techniques deployed to achieve presence/absence detection and these techniques are generally chosen based on the intended application. In "Moving Object Detection Using FPGA"[2] we see that not only did Mr Pawaskar and associates utilize the background subtraction method, they also implemented it using an FPGA. They were able to demonstrate that the background subtraction method is not only capable of producing a sensitive system which can be used to pick up small alterations between the present frame and a reference image but it is also capable of providing the accuracy required to determine the general type of object that is now present in the field of vision.

These findings fully support the initial assumption made in this paper which stated that the background subtraction method in conjunction with an FPGA can indeed be used to provide the accuracy required to detect whether or not objects are present or absent in the manufacturing industry, such as if being passed on a production line. The journal [2] went on to further support the propositions made in this paper which stated that the use of an FPGA will ultimately be a cost-effective and energy saving decision. This is because by nature, an FPGA is a very powerful and cheap tool which has the processing power required to provide the operator with feedback on the presence/absence of the object in a timely manner.

\subsection{Proposed Setup}

So far in the methodology we have discussed how the background subtraction method will be implemented. Now, we shall discuss the proposed setup for the overall flow of the system.

Firstly, after the frames are extracted from the video, we will create a header file for it as well as for the background image which was previously stored. Using Xilinx Platform 
Studio, which is a tool used to link a program to hardware like the FPGA board, we are able to write the algorithm for the back ground subtraction method using $\mathrm{C}$ language. We will input the header file and the $\mathrm{C}$ file into the XPS tool. The bitstream can then be downloaded onto the FPGA for processing to occur and the presence/absence of the object can be determined. The FPGA then indicates the result of the processing and the controller will act accordingly. [2] Figure 2 below illustrates the overall process the system will go through from obtaining the image to notifying the controller of whether or not an object is present.

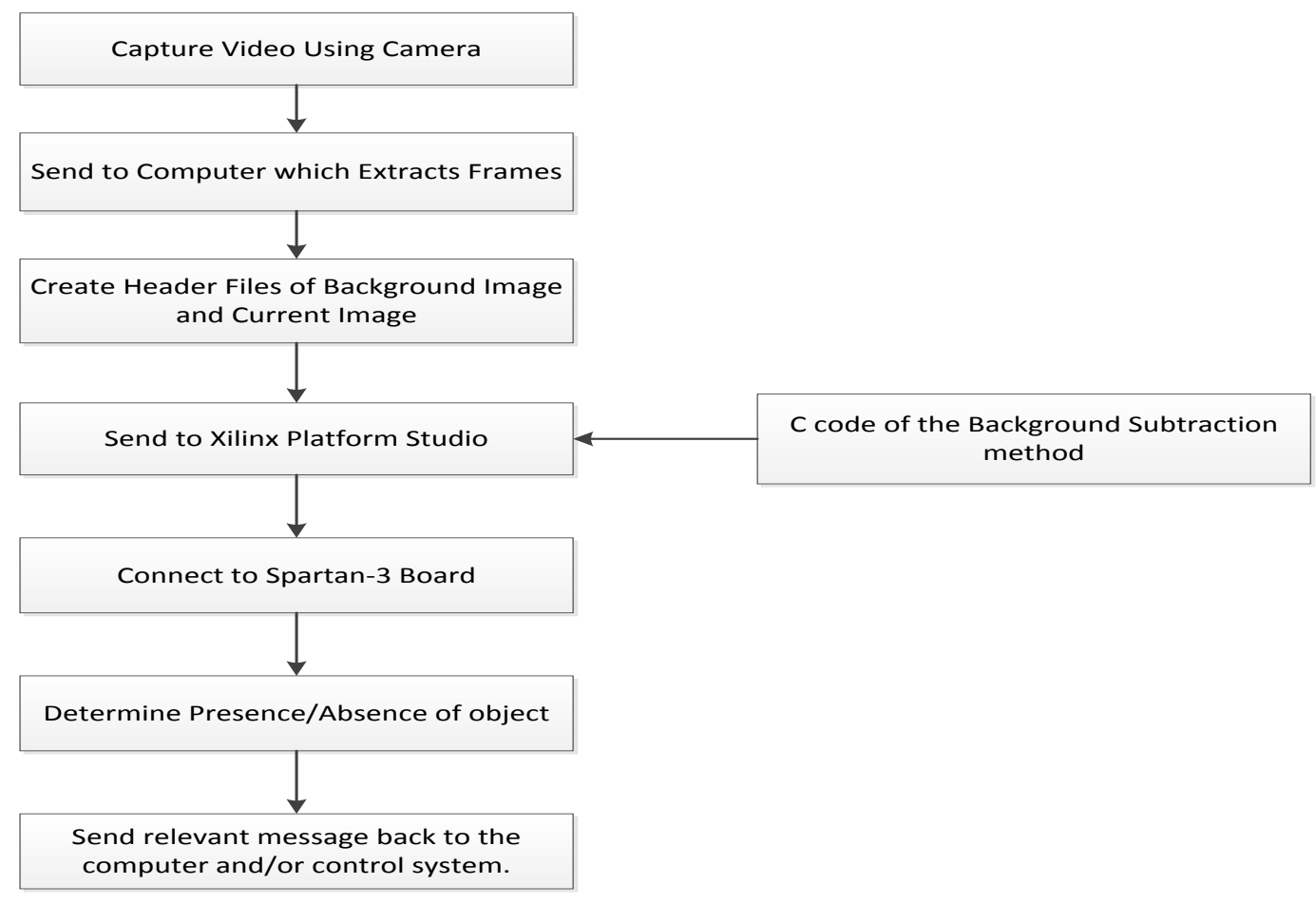

Figure 2. Proposed Process Flow

\section{Conclusion}

This paper proposed the use of an FPGA to implement the background subtraction algorithm to determine the presence/absence of objects in the manufacturing industry. A conceptual diagram was constructed which illustrated the logical flow of steps which can be undertaken to achieve the task as well as it suggested some preferred hardware that can be used. The concept behind the background subtraction method was analysed and it was verified that it is indeed a suitable algorithm to be used as part of a system that can ultimately be implemented in the manufacturing industry. This is because in the industry, the background or space behind the objects that move along the conveyor belt is generally constant, thereby making it possible to detect whether or not an object is present by comparing a frame at some time, $t$, to a reference background image. The fact that the system can be implemented under strict time constraints also shows that the FPGA can indeed be used to increase the speed at which objects can be detected whilst maintaining its accuracy and reliability due to its architecture. Generally, when the ratio of performance per power/hardware resource needs to be maximized, the Field Programmable Gate Array platform is usually the preferred choice [6]. 


\section{References}

[1] C. S'anchez-Ferreira, J. Y. Mori, C. H. Llanos "Background Subtraction Algorithm for Moving Object Detection in FPGA" IEEE (2012).

[2] M. Pawaskar, N. Narkhede and S. Athalye, "Moving Object Detection Using FPGA", International Journal of Emerging Trends \& Technology in Computer Science (IJETTCS), vol. 3, no. 3, (2014).

[3] M. Salve, M. Rajput and S. Shingate, "Fpga Based Moving Object Detection Algorithm Implementation for Traffic Surveillance", Int. Journal of Engineering Research and Applications, vol. 3, no. 5, (2013), pp. 1829-1832,.

[4] M. Chakorkar and M. Patil, "Motion Detection by Background Subtraction Algorithm in FPGA", IOSRJECE, vol. 9, no. 4, (2014), pp. 85-88,.

[5] A. Khule and M. Nagmode, "Parameter Based Presence and Absence Detection for Automated Visual Inspection Applications", International Journal of Science and Research (IJSR), vol. 6, no. 14, (2013).

[6] C. Cheng and C. Bouganis, "An FPGA-based object detector with dynamic workload balancing", 2011 International Conference on Field-Programmable Technology, (2011). 